\title{
Manifestations of phase-coherent transport in graphene
}

\section{The Josephson effect, weak localization, and aperiodic conductance fluctuations}

H.B. Heersche, P. Jarillo-Herrero, J.B. Oostinga, L.M.K. Vandersypen, and A.F. Morpurgo ${ }^{\text {a }}$

Kavli Institute of Nanoscience, Delft University of Technology, PO Box 5046, GA 2600,

Delft, The Netherlands

\begin{abstract}
The electronic transport properties of graphene exhibit pronounced differences from those of conventional two dimensional electron systems investigated in the past. As a consequence, well established phenomena such as the integer quantum Hall effect and weak localization manifest themselves differently in graphene. Here we present an overview of recent experiments that we have performed to probe phase coherent transport. In particular, we have investigated in great detail Josephson supercurrent and superconducting proximity effect in junctions consisting of a graphene layer in between superconducting electrodes. We have also used the same devices to measure aperiodic conductance fluctuations and weak localization. The experimental results clearly indicate that lowtemperature transport in graphene is phase coherent on a $\sim 1 \mu \mathrm{m}$ length scale, irrespective of the position of the Fermi level. We discuss the different behavior of Josephson supercurrent and weak localization in terms of the unusual properties of the electronic states in graphene upon time reversal symmetry.
\end{abstract}

\section{Introduction}

Graphene - a single layer of graphite - is a recently discovered form of carbon that hosts a new kind of two dimensional electron system (2DES) [1-3]. In graphene, the low energy electronic states can be described by two sets of two-dimensional spinors associated to two independent points ( $\mathrm{K}$ and $\mathrm{K}^{\prime}$ ) at the corner of the Brillouin zone. These spinors, whose components describe the amplitude of the wavefunction on the two atoms of unit cell of graphene (the so-called $A$ and $B$ atoms), satisfy the two dimensional Dirac equation for massless particles, with the Fermi velocity $v_{F}$ playing the role of the speed of light $c$.

The first experimental investigations of magneto-transport in graphene have resulted in a number of fascinating discoveries. The quantum Hall effect, for instance, exhibits a new quantization sequence characteristic of relativistic electrons $[2,3]$. In addition, the conductivity remains finite even when the Fermi level is tuned to be at the charge neutrality (or Dirac) point where conduction and valence band touch. This is surprising since at the Dirac point the density of states as well as the density of charge carriers are expected to be vanishingly small. These observations have posed fundamental questions as to the nature of transport in a 2DES of relativistic electrons, and stimulated an impressive amount of theoretical work.

Here we present an overview of experiments that we have performed in the course of the last year to investigate phase coherent transport in graphene. This work was motivated, at least in

\footnotetext{
a e-mail: a.morpurgo@tnw.tudelft.nl
} 
part, by previous studies of weak-localization and aperiodic conductance fluctuations that have resulted in unexpected observations. In particular, in graphene, the low-field magnetoresistance associated to weak-localization has been found to be strongly sample dependent and to be entirely absent in many samples $[4,5]$. This is striking, since this low-field magnetoresistance is a ubiquitous, very reproducible phenomenon, observed in all conventional 2DESs, and understood theoretically in great detail. Furthermore, in these experiments, aperiodic conductance fluctuation due to the random interference of electron waves have been observed only when the Fermi level was tuned to be deep in the conduction or valence band, but not in the vicinity of the Dirac point. This poses the question of whether phase coherent transport is at all possible for electrons at the Dirac point.

Our work focuses on mesoscopic samples, with dimensions smaller than the phase coherence length. To probe the phase coherent propagation of electrons in graphene we have investigated three different phenomena: the Josephson effect in devices with superconducting contacts, lowfield magnetoresistance due to weak-localization, as well as magnetic-field and gate-voltage induced aperiodic conductance fluctuations. These different phenomena probe different aspects of electron dynamics in graphene, which makes their comparison particularly interesting. Specifically, whereas supercurrent and weak-localization are phenomena that require the presence of time reversal symmetry, the occurrence of aperiodic conductance fluctuations only requires transport to be phase coherent. In addition, as we will discuss, the presence of two distinct $\mathrm{K}$-points in graphene plays a different role in determining the behavior of supercurrent and weak localization.

The paper is structured as follows. In section 2 we briefly discuss the sample fabrication and basic characterization by high field magneto-transport measurements. Section 3 focuses on manifestations of phase coherent transport: the measurements of supercurrent (3.1), when the electrodes are in their superconducting state, and study of quantum interference phenomena in the normal transport regime (3.2). Finally, section 4 is devoted to the interpretation of the experimental results and to the discussion of their significance.

\section{Sample fabrication and characterization}

The fabrication of graphene devices is based on the technique developed by Novoselov et al. [1]. It relies on the mechanical exfoliation of thin graphite layers from crystalline graphite powder, by means of an adhesive tape. The tape covered with exfoliated graphite is then brought into contact with a substrate consisting of a degenerately doped silicon substrate (that is used as a gate electrode) covered by a $300 \mathrm{~nm} \mathrm{SiO}_{2}$, onto which a large array of electron beam lithography markers has been previously defined. The graphite layers are thus transferred onto the substrate. Inspection with an optical microscope permits to identify the thinnest layers, that are only faintly visible, including graphene single layers. From the optical microscope images the position of these layers is registered with respect to the markers, to align metal electrodes that are fabricated subsequently by means of electron beam lithography, metal evaporation, and lift off. Figure 1 shows an image of one of our samples fabricated in this way.

We found that the size of the thinnest layers that are transferred onto the substrate depends on the procedure used to exfoliate graphite. In particular, if a larger amount of crystalline graphite powder is randomly placed onto the adhesive tape, and exfoliated many times, only a few, small (typically $\simeq 1 \times 1 \mu \mathrm{m}^{2}$ ) layers are found in the end. All the experiments described in this paper have been performed on graphene flakes prepared in this way. More recently we have found that better results are obtained if only one graphite crystal with the largest possible size (linear dimensions of the order of a few millimeters) and sharp facets is selected under the optical microscope, and exfoliated the least possible number of times to achieve a uniform coverage of the adhesive tape. In this case, many large thin flakes are obtained reproducibly with linear dimensions in excess of $10 \times 10 \mu \mathrm{m}^{2}$ (figure 1 shows one of the largest graphene flakes that we have observed until now and has dimensions of about $30 \times 10 \mu \mathrm{m}^{2}$.) Furthermore we have also found that inspection under the microscope is facilitated by using a camera connected to a computer with a software enabling real-time contrast enhancement. The larger density of 
a

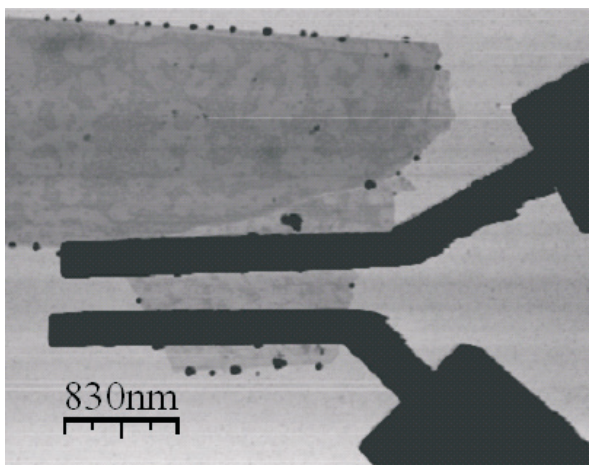

b

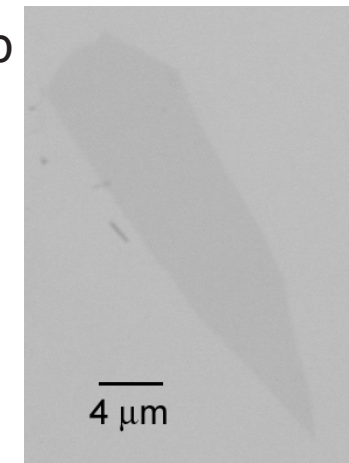

Fig. 1. (a) Atomic force microscopy image of a single-layer graphene Josephson junction used in our experiments. The electrodes consist of a $\mathrm{Ti} / \mathrm{Al}$ bilayer, with the Titanium in contact with graphene. (b) Large graphene layer deposited on top of a $\mathrm{Si} / \mathrm{SiO}_{2}$ substrate by controlled exfoliation of a single graphite crystal.

thin graphite flakes obtained by a careful selection of the starting graphite crystal and the use of a contrast-enhancing software for the detection of thin layers drastically expedite the device fabrication process.

Since the original goal of our work was the study of supercurrent through graphene, all our devices consisted of a thin graphite layer contacted by closely spaced $(\simeq 200$ to $500 \mathrm{~nm})$ superconducting electrodes. As superconducting material we used a Ti/Al bilayer with Titanium in contact with graphene. The use of Titanium results in highly transparent contacts and the top Aluminum layer enhances the critical temperature to about $\simeq 1 \mathrm{~K}$, much higher than the lowest temperature accessible in our dilution refrigerator $(\simeq 20 \mathrm{mK})$. In all flakes investigated we have observed large changes in the conductance upon the application of a voltage to the gate electrode (see figure 2), indicating that the flakes indeed consist of a small number of layers.

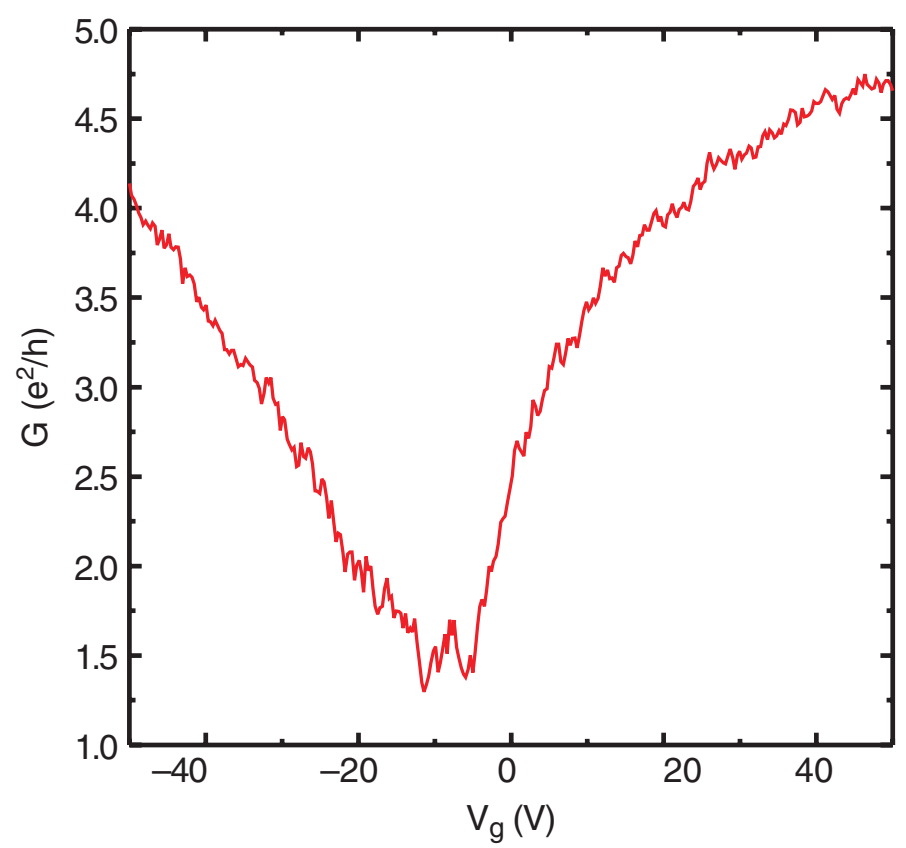

Fig. 2. Two-terminal conductance $G$ as a function of gate voltage $V_{G}$ measured at $B=0 \mathrm{~T}$ and $T=20 \mathrm{mK}$. The position of the minimum conductance corresponds to gate voltage $\left(V_{G}=V_{D}\right)$ for which the Fermi level is located at the Dirac point. 


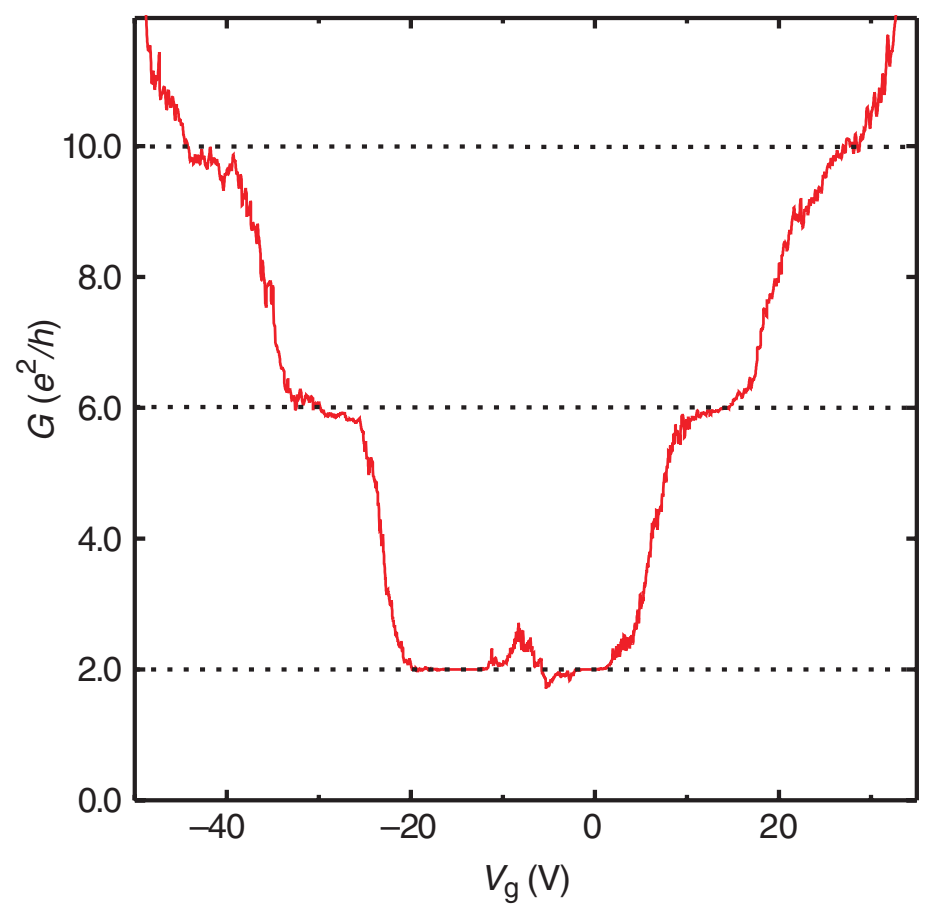

Fig. 3. Two terminal conductance $G$ as a function of gate voltage $V_{G}$ measured in the presence of high magnetic field $(B=12 \mathrm{~T})$ at $T=20 \mathrm{mK}$. Conductance plateaus at $2,6,10 \ldots, e^{2} / \mathrm{h}$ are clearly apparent, in agreement with the quantization values expected for a single graphene layer.

To identify single graphene layers we have performed high-field magnetotransport measurements. Even in the two terminal geometry of our superconducting junctions clear plateaus in the conductance can be observed (see figure 3), which originate from the formation of quantum Hall edge channels. It is apparent that the conductance values at which the plateaus appear satisfy the quantization sequence expected for a single graphene layer. In this way, we could successfully identify four of our samples to consist of an individual graphene layer; the remaining samples could not be identified unambiguously and consist probably of stacks of two to four graphene layers. In the following section we only show the results of measurements taken on single-layer devices.

\section{Manifestations of phase coherent transport}

\subsection{Josephson effect}

The phenomenon that is most commonly studied to investigate phase coherent transport in normal conductors is weak-localization, which manifests itself in the suppression of the resistivity upon the application of a small magnetic field. In graphene, first investigations of weak-localization have shown signals of different magnitude in different samples [4,5]. More strikingly, the magnitude of the weak localization signal was found to be often suppressed or even vanishing. In order to understand this unusual behavior, it is useful to investigate other phenomena that rely on the presence of phase coherence. In this regard, the study of supercurrent in a Josephson junction consisting of a graphene layer in between two superconducting electrodes is an excellent candidate. In fact, the Josephson effect does not only depend on transport being phase coherent, but, similarly to weak-localization, it also requires the system to possess time reversal symmetry [6].

Figure 4 shows the current-voltage (I-V) characteristics of a single layer device measured at the base temperature of our dilution refrigerator $(T \simeq 20 \mathrm{mK})$. The current flows without 


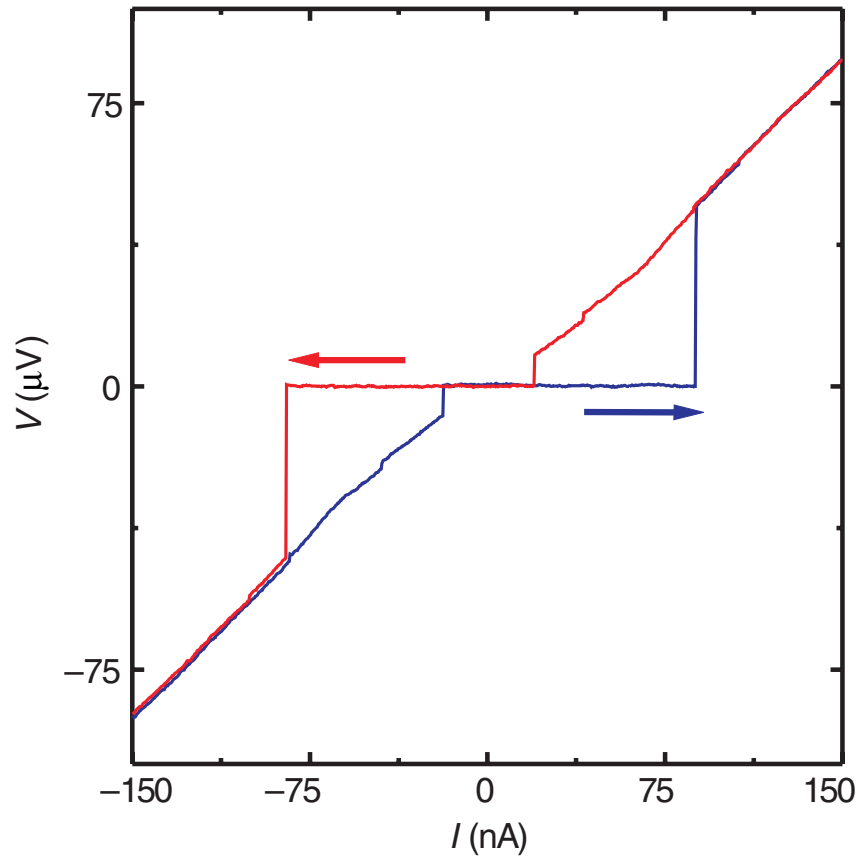

Fig. 4. Volt-Amperometric $(I-V)$ characteristics of one of the graphene Josephson junctions that we have investigated, exhibiting a clear supercurrent. The measurements have been performed at $T=20 \mathrm{mK}$; the arrows indicate the direction in which the current is swept during the measurement.

resistance (no voltage drop at finite current) below the critical current, $I_{c}$. In different devices, $I_{c}$ ranged from a few tens of nanoamperes to almost 1 microampere, depending on the gate voltage and on the geometrical dimensions of the junctions. The typical device width was $\simeq 1 \mu \mathrm{m}$ and contact separations between 200 and $500 \mathrm{~nm}$ were studied. Note, in passing, that what we actually measure is the switching current; the intrinsic $I_{c}$ may be higher $[6,7]$. Remarkably, we have measured proximity-induced supercurrents in all the devices that we tested - 17 flakes in total, with several devices on some flakes - four of which were unambiguously identified as single layer graphene via the quantum Hall effect. The systematic presence of a supercurrent demonstrates the robustness of the Josephson effect in graphene junctions.

To further investigate the superconducting properties of our devices we measured the dependence of $I_{c}$ on magnetic field (figure 5). The critical current exhibits an oscillatory Fraunhoferlike pattern, with at least 6 visible side lobes, which is indicative of a uniform supercurrent density distribution [8]. The periodicity of the oscillations is theoretically expected to be approximately equal to a flux quantum $\Phi_{0}$ divided by the junction area. The area that corresponds to the $2.5 \pm 0.5 \mathrm{mT}$ period is $0.8 \pm 0.2 \mu \mathrm{m}^{2}$, which is in good agreement with the measured device area $\left(0.7 \pm 0.2 \mu \mathrm{m}^{2}\right)$ determined from the AFM image.

Having established the existence of the Josephson effect in graphene, we analyse the gate voltage dependence of the critical current. Figure 6 shows several $I$ - $V$ traces taken at different $V_{G}$, where it can be seen that varying the gate voltage has a strong effect on the maximum supercurrent flowing through the device. This behaviour can be more readily seen in figure 7 , where the differential resistance is plotted as a function of current bias and gate voltage. By changing $V_{G}$ we can shift continuously the Fermi energy from the valence band $\left(V_{G}<V_{D}\right)$ to the conduction band $\left(V_{G}>V_{D}\right)$ : irrespective of the sign of $V_{G}$, we find a finite supercurrent. This demonstrates that the devices operate as bipolar supercurrent transistors: the supercurrent is carried by hole Cooper pairs when the Fermi level is in the valence band and by electron Cooper pairs when it is in the conduction band. Note that in going from valence to conduction band, we sweep the position of the Fermi level through the Dirac point. Strikingly, even then the supercurrent remains finite, despite the fact that for perfect graphene theory predicts a vanishing density of states at $V_{G}=V_{D}$ [9]. This behaviour has been observed in all samples and demonstrates that electronic transport in graphene is phase coherent irrespective of the gate voltage, including when the Fermi energy is located at the Dirac point. 


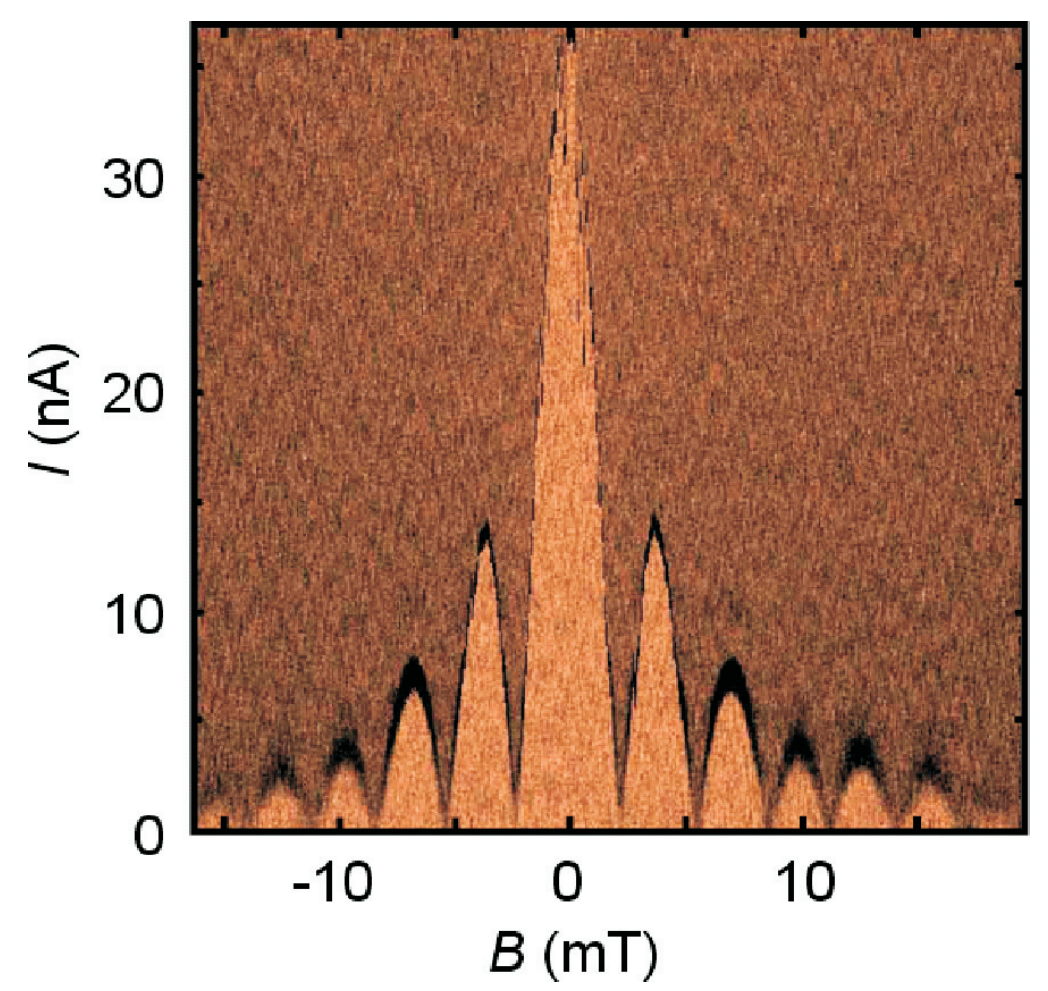

Fig. 5. Color-scale representation of the differential resistance measured as a function of current bias and magnetic field $(d V / d I(I, B))$ at $T=20 \mathrm{mK}$ (the light color corresponds to zero differential resistance, i.e. the junction is in the superconducting state. Dark color corresponds to finite $d V / d I)$. The critical current exhibits a series of oscillations typical of a Fraunhofer pattern. The presence of many lobes of decreasing amplitude indicates that the supercurrent density is rather uniformly distributed across the junction.

In conventional Josephson junctions, the critical current correlates with the normal state conductance, $G_{n}[10]$. In graphene this correlation can be observed directly, as shown in figure 7, because both $I_{c}$ and $G_{n}$ depend on $V_{G}$. To analyse this correlation, we plot the product of the measured critical current and the normal state resistance $\left(R_{n}=1 / G_{n}\right)$, or $I_{c} R_{n}$ product (see figure 8). We find that at high gate voltage $I_{c} R_{n} \approx \Delta / e$, and that $I_{c} R_{n}$ is suppressed by a factor $2-3$ around the Dirac point. The observed $I_{c} R_{n}$-product is thus close to the theoretical value of $\sim 2.5 \Delta / e$ for a model system of graphene in the ballistic regime [11]. The remaining discrepancy could be easily accounted for by the difference between the measured critical current (or switching current) and the intrinsic critical current [6,7]. This difference is in general hard to quantify and depends on the electromagnetic environment of the sample, but it is expected to be more pronounced when $I_{c}$ itself is smaller (i.e. at low $V_{G}$ ), and could thus well explain the observed $V_{G}$ dependence of $I_{c} R_{n}$.

\subsection{Normal state transport}

Next to the study of superconducting proximity effect, we have measured transport in our devices at temperature higher than the critical temperature of Aluminum $(\approx 1.3 \mathrm{~K})$. With the sample dimensions being smaller than the phase coherence length in graphene, we expect the devices to exhibit all the characteristic signatures of mesoscopic transport, i.e. aperiodic conductance fluctuations as a function of magnetic field and gate voltage and, upon ensemble averaging, weak localization [12].

Figure 9 shows the magnetoresistance of one of our devices for different values of gate voltage in the range $(-6 \mathrm{~V},-7.5 \mathrm{~V})$, which corresponds to sweeping the Fermi level across the 


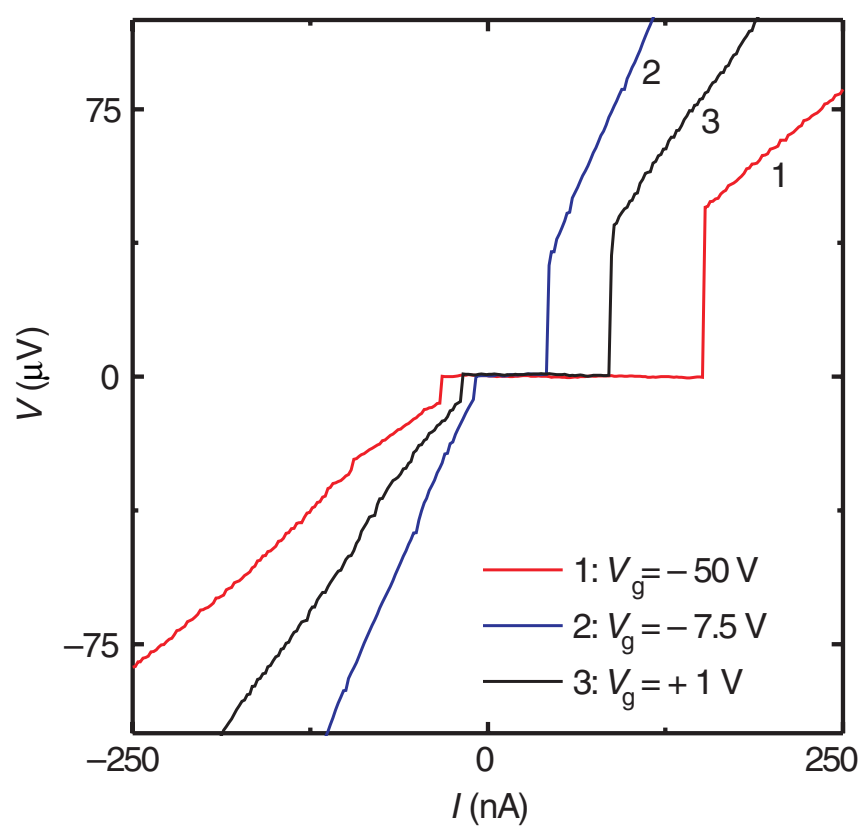

Fig. 6. Volt-Amperometric $(I-V)$ characteristics measured at $T=$ $20 \mathrm{mK}$ for different values of gate voltage (as indicated in the legend). The current bias is swept from negative to positive values.

Dirac point. The presence of aperiodic conductance fluctuations is apparent. The characteristic correlation field is approximately $5-10 \mathrm{mT}$, that compares well to the expected value of $h / e S$ (where $\mathrm{S}$ is the area of the device). At this temperature $(T=1.3 \mathrm{~K})$, the root mean square amplitude of these fluctuations is smaller than $e^{2} / h$, but we also observed that the amplitude of the aperiodic conductance fluctuations increases with lowering $T$ (at $T=20 \mathrm{mK}$, we measured aperiodic conductance fluctuations by applying a magnetic field larger than the critical field of the superconducting electrodes), resulting in root mean square values $\simeq e^{2} / h$ at $T=20 \mathrm{mK}$. Similar measurements performed in a few different samples gave comparable, consistent results. Although a more systematic study as a function of gate voltage and temperature remains to be done, the results of our experiments allow us to conclude that aperiodic conductance fluctuations are indeed present in graphene even when the Fermi level is at or near the Dirac point, and that their magnitude is close to what one would expect for conventional mesoscopic conductors.

In order to measure the signal due to weak localization we have performed an average of the magnetoresistance traces measured at the different values of gate voltage. This procedure is commonly used in conventional mesoscopic systems such as open quantum dots [13]. It is equivalent to take an "ensemble averaged" and is justified as long as the average device properties do not change significantly in the gate voltage range investigated. For graphene this is important and should be noted explicitly, because in the neighborhood of the Dirac point the dependence of the device conductance on $V_{G}$ is, a priori, non negligible.

Figure 10 shows the ensemble averaged, low-field magnetoconductance of two different devices exhibiting a clear conductance minimum at $B=0$, originating from weak-localization. This clearly shows that weak-localization is also present in graphene, even in the neighborhood of the Dirac point. Contrary to the case of aperiodic conductance fluctuations, however, the magnitude of the magnetoconductance due to weak localization is rather different in different samples, and often considerably smaller than $e^{2} / h$ (we found values ranging from 0.1 to 0.5 $e^{2} / h$ in different samples). This observation is consistent with results obtained by others and confirms that the occurrence of weak-localization in graphene is surprisingly sample dependent.

We have also performed similar measurements at high gate voltage (i.e. away from the Dirac point) and found that both aperiodic conductance fluctuations and weak localization are present there as well. More systematic measurements would be of interest to determine the precise gate voltage dependence, as well as the full temperature dependence, of weak localization. Such studies have not yet been performed. 


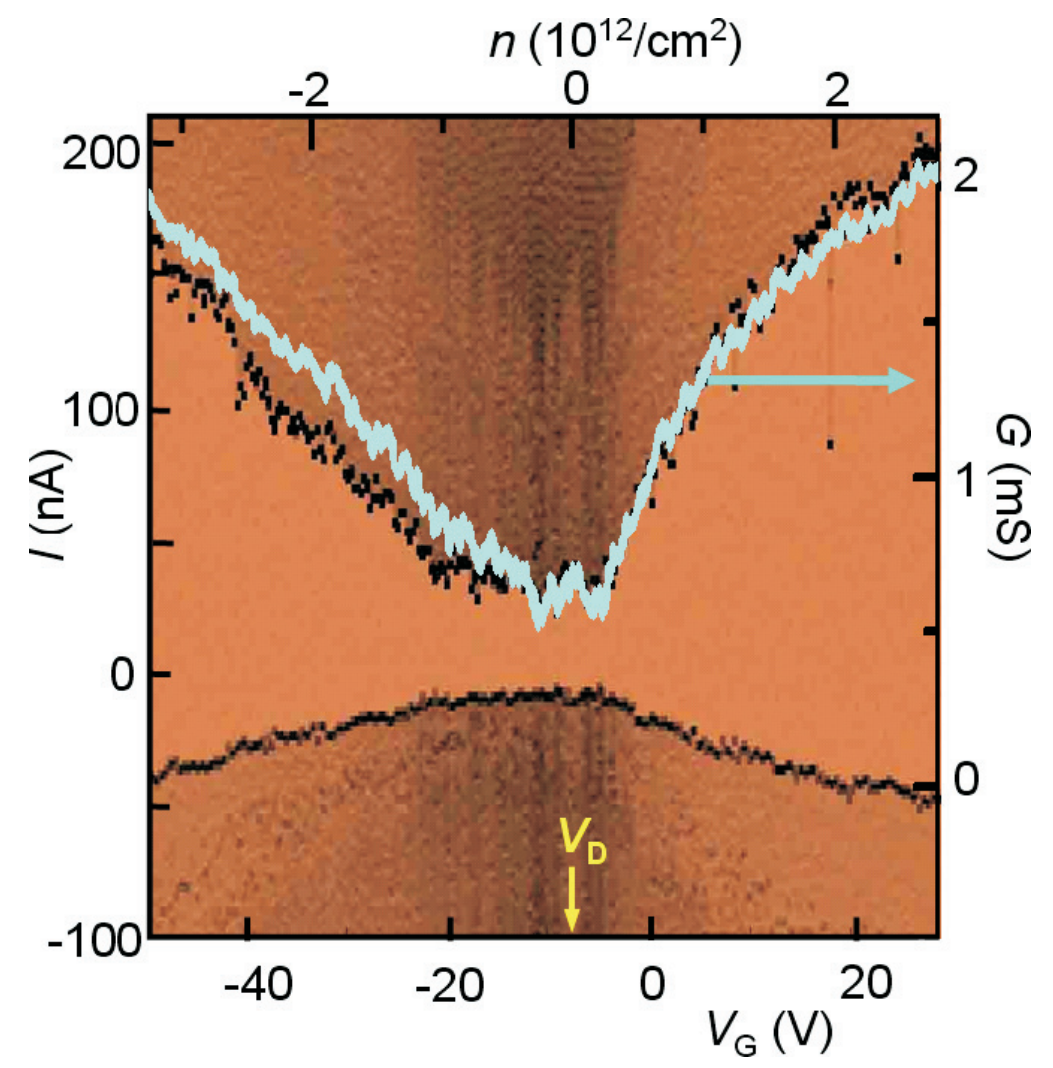

Fig. 7. Colour-scale plot of the differential resistance measured in one of our Josephson junctions, as a function of current and gate voltage. For all values of gate voltages, the measurements have been taken sweeping the current from negative to positive values, which explains the apparent asymmetry in critical current (see also figure 4). The lighter color corresponds to $d V / d I=0$, i.e., the junction is in the superconducting region. The top axis shows the electron density, $n$, as obtained from the known capacitance to the gate electrode. Note that for large negative (positive) $V_{G}$ the supercurrent is carried by holes (electrons), i.e. our devices function as a bipolar Josephson transistor. Note also that the supercurrent at the Dirac point is finite. The light blue curve is the normal state conductance of the junction, whose gate voltage dependence correlates well with that of the critical current.

\section{Discussion}

The comparative study of Josephson supercurrent, weak localization, and aperiodic conductance fluctuations permits to draw conclusions about two important aspects of the electronic properties of graphene, namely the phase coherent nature of transport and the role of time reversal symmetry in electronic transport. All our observations consistently point to the fact that transport in graphene is phase coherent irrespective of the position of the Fermi level, i.e. whether the Fermi level is near or far away from the Dirac point. From the observation of a finite supercurrent in devices where the electrode spacing was $500 \mathrm{~nm}$, we can also conclude that the phase coherence length is at least $500 \mathrm{~nm}$ at $20 \mathrm{mK}$. What is particularly interesting and deserves a special discussion, however, is the difference in the behavior of the supercurrent and weak localization. More specifically, the question is why supercurrent is present in all devices investigated whereas the magnitude of the weak-localization correction exhibits much larger differences in different samples [5]. As both the presence of Josephson supercurrent and the occurrence of weak localization require the system to be time reversal invariant, it is useful to analyze the symmetry properties of graphene with respect to time reversal transformations.

In graphene, the symmetry properties upon time reversal transformation are related to the peculiar electronic structure of this material. At low energy, the band structure of graphene 


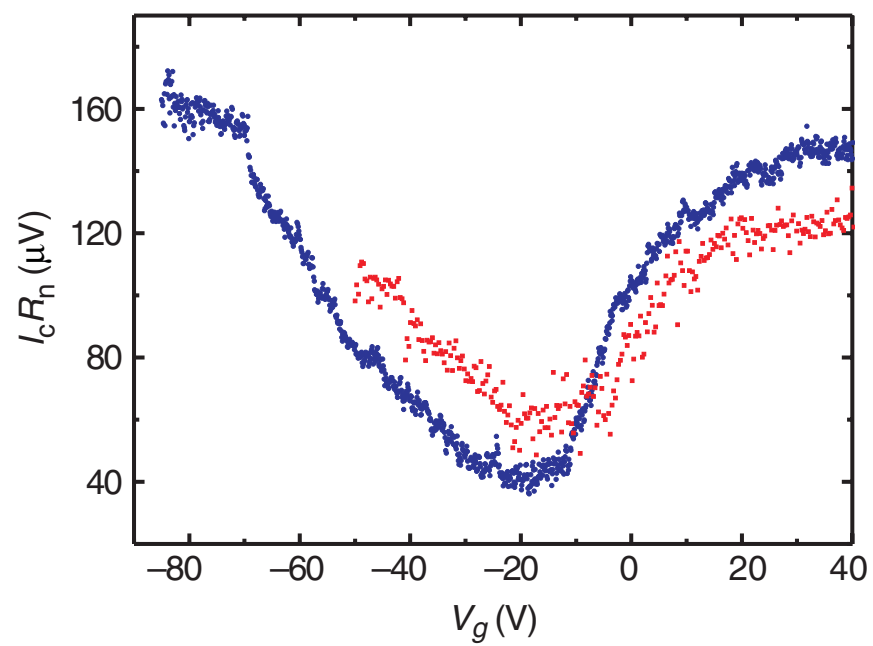

Fig. 8. Product of critical current and normal state resistance (i.e., $I_{c} R_{N}$-product) measured at $T=$ $20 \mathrm{mK}$, as a function of gate voltage $V_{G}$ for two different samples. Although $I_{c} R_{n}$ remains of the order of $\Delta / e$ over the entire gate voltage range, a suppression is always observed around the Dirac point.

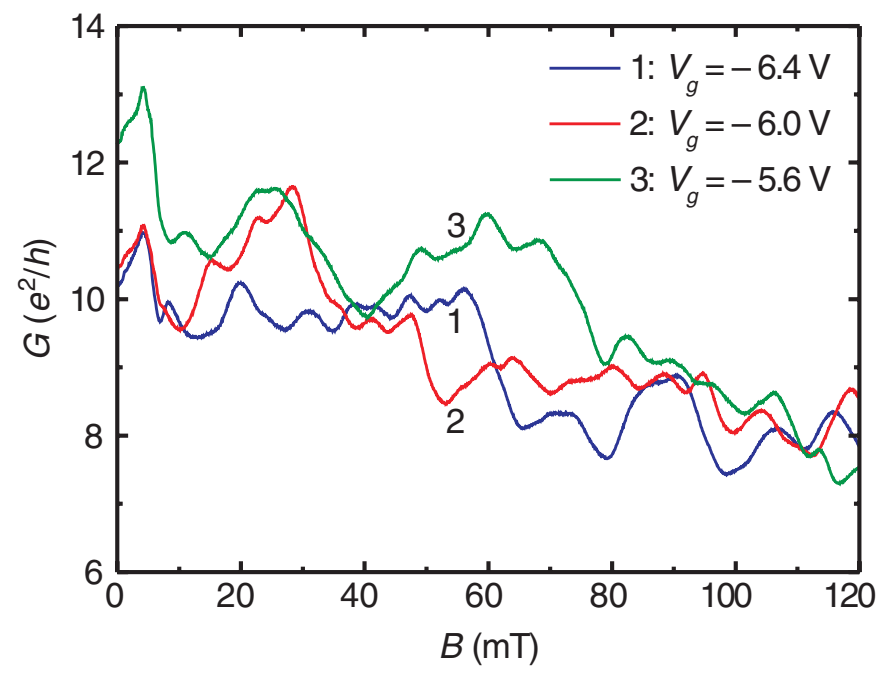

Fig. 9. Two-terminal magnetoconductance measured at $1.6 \mathrm{~K}$, for small values of the applied magnetic field $B$, exhibiting aperiodic conductance fluctuations for different values of the gate voltage near the Dirac point.

can be described in terms of two identical, independent valleys centered on the so-called $\mathrm{K}$ and $\mathrm{K}^{\prime}$ points [9]. Upon time reversal, states in one of the valleys are transformed into states belonging to the opposite valley. As a consequence, the manifestation of phenomena that rely on the presence of time reversal symmetry depends critically on whether mechanisms are present that can couple states in the two valleys. In simple terms, if the two valleys are perfectly decoupled, an electron propagating in one of the valleys can only occupy a subset of all the possible quantum states, which does not include time reversed states. As a consequence the electrons behave as if time reversal symmetry in the system was absent.

This conclusion has important consequences for quantum interference, since it implies that when the two valleys are fully decoupled, interference originating from coherent propagation of electrons along time reversed trajectories cannot occur. Therefore quantum corrections to the conductivity, like weak localization, would be absent (see, however, appendix A for an additional symmetry present in graphene that may result in a quantum correction of the conductivity in graphene). The contribution of time-reversed trajectories to quantum interference can be restored only up to the extent that there exists a coupling mechanism between the two valleys. In the absence of superconducting electrodes, coupling between the valleys is caused by extrinsic 


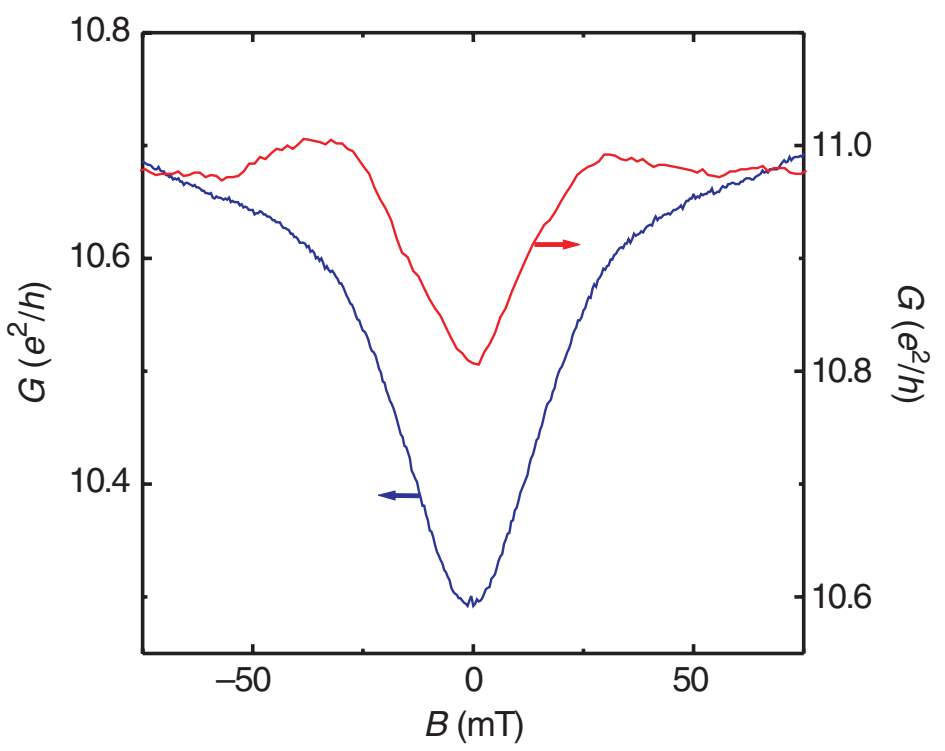

Fig. 10. Ensemble averaged magnetoconductance measured at $T=1.6 \mathrm{~K}$ in two different devices. In both cases, the average has been taken on approximately 60 different traces each measured at a different value of gate voltage, within a few volts on either side of the Dirac point.

mechanisms such as short-range scattering at impurities or at the graphene edges [14-16]. This makes the occurrence of weak localization dependent on extrinsic defects, which explains why both in our own samples (see figure 10) and other recent measurements $[4,17]$ weak localization is found to exhibit a surprising sample dependent behaviour and is often suppressed.

The situation is different in the presence of superconducting electrodes. Since Cooper pairs are made out of time reversed electron states, the two electrons in Cooper pairs that are injected from the superconducting electrodes into graphene go to opposite K-points [11]. In this way, the presence of a superconducting electrode provides an intrinsic mechanism that couples phase coherently electronic states belonging to opposite valleys. Because of this coupling, the dynamics of electrons in the presence of superconducting electrodes is then described by the full (twovalley) Hamiltonian of graphene that is time-reversal symmetric. We believe that this is why, contrary to weak localization, a supercurrent has been observed in all the devices that we have investigated.

\section{Appendix A}

Even in the absence of coupling within the two $\mathrm{K}$ points it is in principle possible to have quantum correction to the conductivity in graphene, because of an additional symmetry which is present in the long wavelength approximation, and that operates within one single valley. This symmetry, however, is rather fragile and it is broken by a number of different physical perturbations. For this reason, the experimental relevance of this symmetry for current graphene devices is unclear.

To understand this point in more detail, let us consider the effective Hamiltonian for one $\mathrm{K}$-point in the long-wavelength approximation, which is a $2 \times 2$ matrix acting on the components of the wavefunction on the two independent sublattices of graphene (the so-called $A$ and $B$ atoms). It reads

$$
H_{K}=\hbar v_{F}\left(\begin{array}{cc}
0 & k_{x}-i k_{y} \\
k_{x}+i k_{y} & 0
\end{array}\right)
$$

with $\mathbf{k}=\left(k_{x}, k_{y}\right)$ the momentum of the electron measured relative to the K-point. This $2 \times 2$ matrix is left invariant by the anti-unitary transformation $U=i \sigma_{y} \widehat{K}$, i.e. $U^{\dagger} H_{K} U=H_{K}$, 
where $\sigma_{y}$ is the usual Pauli matrix and $\widehat{K}$ denotes complex conjugation. $U$ is the usual operator associated to the time reversal symmetry transformation for spin $1 / 2$ particles. In this sense, the operator $U$ defines an "effective" time reversal symmetry transformation that is applicable in a single valley only and reverses the sign of the momentum relative to the K-point. In the presence of certain types of smooth scattering potentials (see [15]), there exist "effectively" time-reversed trajectories along which the electrons can propagate while remaining within the same valley. On this basis, Suzuura and Ando [15] predicted that these trajectories cause a quantum correction to the conductivity in the form of weak antilocalization.

The operator $U$ however does not represent the true time reversal symmetry transformation for electrons in graphene, as true TRS connects states belonging to the two opposite K-points in graphene and cannot be described within the single K-point approximation. For this reason the effective TRS represented by $U$ is not robust against physical perturbations that normally preserve TRS (e.g. static potentials). For instance, effective TRS is broken by (i) curvature in graphene $[4,14]$ (that can be described within a single K-point approximation as a vector potential), (ii) scattering at edges [18], and (iii) trigonal warping [16] (i.e., by including quadratic terms in the long wavelength expansion from which the one K-point Hamiltonian is obtained). As a consequence, in real experimental samples the observation of effects associated to the symmetry $U$ is expected to be very difficult (at least in samples of current quality).

\section{References}

1. K.S. Novoselov et al., Science 306, 666 (2004)

2. K.S. Novoselov et al., Nature 438, 197 (2005)

3. Y.B. Zhang, Y.W. Tan, H.L. Stormer, P. Kim, Nature 438, 201 (2005)

4. S.V. Morozov et al., Phys. Rev. Lett. 97, 016801 (2006)

5. C. Berger et al., Science 312, 1191 (2006)

6. M. Tinkham, Introduction to Superconductivity (McGraw-Hill, Singapore, 1996)

7. P. Joyez, P. Lafarge, A. Filipe, D. Esteve, M.H. Devoret, Phys. Rev. Lett. 72, 2458 (1994)

8. A. Barone, G. Paterno, Physics and Applications of the Josephson Effect (John Wiley \& Sons, New York, 1982)

9. M.S. Dresselhaus, G. Dresselhaus, P.C. Eklund, Science of Fullerenes and Carbon Nanotubes (Academic Press, San Diego, 1996)

10. K.K. Likharev, Rev. Mod. Phys. 51, 101 (1979)

11. M. Titov, C.W.J. Beenakker, Phys. Rev. B 74, 041401(R) (2006)

12. B.L. Altshuler, P.A. Lee, R.A. Webb, Mesoscopic Phenomena in Solids (North-Holland, Amsterdam, 1991)

13. J.A. Folk et al., Phys. Rev. Lett. 76, 1699 (1996)

14. A.F. Morpurgo, F. Guinea, Phys. Rev. Lett. 97, 196804 (2006)

15. H. Suzuura, T. Ando, Phys. Rev. Lett. 89, 266603 (2002)

16. E. McCann et al., Phys. Rev. Lett. 97, 146805 (2006)

17. S. Datta, Electronic Transport in Mesoscopic Systems (Cambridge University Press, Cambridge, 1995)

18. M.V. Berry, R.J. Mondragon, Proc. R. Soc. Lond. A 412, 53 (1987) 